\title{
Perception of a Naturalistic Stressor Interacts with 5-HTTLPR/rs25531 Genotype and Gender to Impact Reward Responsiveness
}

\author{
Yuliya Nikolova ${ }^{a}$ Ryan Bogdan ${ }^{a}$ Diego A. Pizzagallia, b \\ ${ }^{a}$ Affective Neuroscience Laboratory, Department of Psychology, Harvard University, Cambridge, Mass., and \\ ${ }^{\mathrm{b}}$ Center for Depression, Anxiety and Stress Research and Neuroimaging Center, McLean Hospital and Harvard \\ Medical School, Belmont, Mass., USA
}

\section{Key Words}

5-HTTLPR · Anhedonia · Depression · Stress · Reward • Resilience

\begin{abstract}
Background: Stressful life experiences frequently precede the onset of major depression; however, the mechanisms that underlie this link are poorly understood. Importantly, some individuals are more susceptible to the depressogenic effects of stress than others. Carriers of the $S$ or $L_{G}$ allele of the 5 -HTTLPR/rs25531 polymorphisms ( $S^{\prime}$ participants) have been found to be more prone to developing depression under stress relative to $L$ or $L_{A}$ homozygotes ( $L^{\prime}$ participants). Moreover, emerging evidence indicates that stress-induced anhedonia may be a mechanism underlying links between stress and depression. Given these findings, we hypothesized that exposure to a naturalistic stressor (school final examinations) would disrupt reward responsiveness (a key behavioral component of anhedonia), and that this effect would be strongest in S' participants. Methods: To objectively assess reward responsiveness, we administered a probabilistic reward task to 70 Bulgarian high school students over two sessions in the 6-month period preceding
\end{abstract}

school finals. For each participant, the two sessions were designated as the 'stress' and 'control' conditions based on self-reported perceived stress. Results: A genotype $\times$ condition interaction emerged in males, with $S^{\prime}$ participants showing larger stress-related reduction in reward responsiveness relative to L' participants. Conclusion: While in need of replication in a larger sample, our results indicate that stress associated with a real-life event is linked to reduced reward responsiveness, the susceptibility to which is modulated by 5-HTTLPR/rs25531 genotype. Although preliminary, these findings identify anhedonia as a promising mechanism linking 5-HTTLPR/rs25531 genotype and stress to depression.

Copyright $\odot 2011$ S. Karger AG, Basel

\section{Introduction}

Epidemiological studies indicate that stressful life events are among the most reliable predictors of the onset and course of major depressive disorder (MDD) ([1-3], for review see [4]); however, the mechanisms underlying this relationship remain incompletely understood. A sizeable body of non-human animal research suggests

\section{KARGER}

(c) 2011 S. Karger AG, Basel

Fax +4161306 1234

E-Mail karger@karger.ch

www.karger.com
Accessible online at:

www.karger.com/nps
Diego A. Pizzagalli

Center for Depression, Anxiety and Stress Research

Room 233C, McLean Hospital

115 Mill Street, Belmont, MA 02478 (USA)

Tel. +1 617855 4230, E-Mail dap@ mclean.harvard.edu 
that stress might partially exert its depressogenic effects by inducing anhedonic-like behavior. Specifically, animal studies indicate that exposure to stressors, in particular chronic and inescapable ones, produces blunted reactivity to natural rewards, as well as dysfunction within mesolimbic dopaminergic pathways critically implicated in incentive motivation and reinforcement learning (for reviews, see $[5,6]$ ).

Emerging evidence suggests that this pattern may translate to humans. An early study focusing on realworld stressors found reduced self-reported hedonic capacity in college students and US Army cadets following stressful final examinations or field training exercises, respectively, and demonstrated that these deficits were more pronounced in participants with a family history of depression [7]. Two recent independent studies demonstrated that acute stress exposure in a laboratory environment (threat-of-shock) resulted in impaired reward learning in a nonclinical sample, particularly in individuals reporting elevated anhedonic symptoms $[8,9]$. Unlike the former study, which employed self-report measures to assess hedonic capacity, these latter studies used a laboratory-based measure of anhedonia to empirically assess participants' ability to modulate their behavior as a function of prior reinforcement history [10]. In addition, using the same laboratory-based reward processing paradigm, Pizzagalli et al. [11] found that healthy participants appraising their daily life in the week prior to testing as stressful, uncontrollable, and overwhelming were similarly characterized by diminished reward learning.

Consistent with these behavioral effects, acute psychosocial stress decreases the responsiveness of a number of limbic structures involved in the experience of pleasure [12]. Finally, early childhood adversity (e.g. abuse, maltreatment) has been associated with elevated levels of anhedonic symptoms in adolescent depression [13] and, perhaps even more strikingly, a blunted response to reward cues in the globus pallidus, a brain region implicated in reward processing, in nondepressed adults [14]. In sum, results from multiple animal experiments and emerging human research spanning a variety of acute and chronic stressors, suggest that stress-related hedonic impairments may, at least partially, account for the depressogenic effects of life stress.

A complete understanding of the association between stress-related anhedonia and depression is critically dependent on an improved delineation of the neurobiological mechanisms underlying reward processing. While dopamine and the opioids are the primary neurotrans- mitters linked to reward processing [15], growing evidence has also implicated serotonin 5-hydroxytryptamine (5-HT). Specifically, genetic knockout of the serotonin transporter (5-HTT) and the resulting 5-HT signaling changes have been found to disrupt the development of the reward system [16] and interfere with reward-based learning [17]. Additionally, pharmacologically elevated 5-HT levels decrease dopamine burst firing in the rat ventral tegmental area [18], thus suppressing neural signals central to reward learning [19]. Taken together, these studies indicate that 5-HT plays an important modulatory role in reward processing.

Among the factors impacting 5-HT signaling in humans is a common functional polymorphism in the promoter region of 5-HTT (5-HTTLPR), resulting in either a short (S) or a long (L) allele of the gene [20]. Importantly, the $S$ allele has been linked to reduced 5-HTT expression relative to the L allele [20]. Furthermore, compared to $\mathrm{L}$ homozygotes, $\mathrm{S}$ carriers are at increased risk of developing depression following stressful life events [21, but see also 22]. Critically, a A/G single nucleotide polymorphism (SNP) within the 5-HTTLPR (rs25531) has been shown to differentially impact $\mathrm{L}$ allele function as well as its clinical association with MDD [23, 24], suggesting that the $\mathrm{L}_{\mathrm{G}}$ allele may be functionally similar to the $\mathrm{S}$ allele. Thus, this SNP may help clarify inconsistent 5-HTTLPR $\times$ Stress interaction results (e.g. [22]). Based on the literature reviewed above, we reasoned that stress-related anhedonia may contribute to links among stress, 5HTTLPR/rs25531 genotype, and depression.

The goal of the present study was to investigate how perceived stress associated with a naturalistic stressor (preparation for important school examinations) and 5-HTTLPR/rs25531 genotype impact reward responsiveness, an objective measure of participants' ability to modulate behavior as a function of previous reinforcement, which has been found to correlate with, and predict, future anhedonic symptoms [10]. In approaching this question, we addressed the limitations of the few human studies that have assessed the role of stress on anhedonia, including reliance on self-report measures of anhedonia [7] and the use of acute laboratory stressors with limited ecological validity [8]. We hypothesized that, relative to L or $\mathrm{L}_{\mathrm{A}}$ homozygotes ('L' participants'), $\mathrm{S}$ or $\mathrm{L}_{\mathrm{G}}$ allele carriers ('S' participants') would show larger stress-related reward responsiveness impairments. Based on research suggesting that gender impacts the nature of 5-HTTLPR $\times$ Stress interactions with regard to depression [25, 26], we also explored the putatively moderating role of gender. 


\section{Methods}

\section{Participants}

Graduating seniors $(\mathrm{n}=116)$ attending high school in Yambol, Bulgaria, completed two study sessions within 6 months of each other. During each session, participants received 20 Bulgarian Leva ( USD 15 ) for their time, and 'won' an additional 13.9515.00 Leva during the task. The Committee on the Use of Human Subjects at Harvard University and the High School Board in Yambol, Bulgaria approved the study. In light of the fact that our aim was to investigate the specific effects of genotype and ongoing stress on reward responsiveness, rather than factors that may affect reward processing and/or vulnerability to depression independently, participants reporting personal or family history of unipolar or bipolar depression $(n=15,12.9 \%)$ and drug use within the month prior to session $2(n=11,9.5 \%)$ were excluded. Ten additional participants $(8.6 \%)$ failed to provide complete information regarding exam-related stress in at least one study session. Another 11 participants' data (9.5\%) were excluded for reaction time outliers and/or poor accuracy on the behavioral task in session $1(n=3)$, session $2(n=3)$, or both sessions $(n=5)$ (see 'Data Reduction and Processing'), leaving a final sample of 70 participants (45\% female, mean age upon study completion: $18.47 \pm$ $0.50)$ available for analyses. Excluded participants did not differ from those included in age, genotype, or gender (all p >0.37).

\section{School Final Examinations}

Beginning in the 2007/2008 academic year, all high-school seniors in Bulgaria are required to take at least two School Final Examinations (SFEs). We chose to use these examinations as a naturally occurring stressor for several reasons: (1) unlike university entrance examinations, these examinations are mandatory for all students, including students who applied to overseas universities or who would not be applying to universities; (2) a passing grade on these examinations is required for high school graduation; (3) grades are printed on students' high school diplomas; (4) results are used in Bulgarian university admission decisions alongside traditional entrance examinations, and (5) the academic year 2007/2008, during which data collection took place, was the first year these tests were administered; accordingly, we expected the uncertainty experienced in the face of novelty to further increase the stress normally associated with taking examinations [27]. The two study sessions occurred 6 months (December 2007) and 2-3 days (May 2008) prior to students' SFEs, respectively.

A Bulgarian sample was chosen instead of an American sample for several theoretical and practical reasons: (1) the population was expected to be relatively genetically homogeneous, which would prevent potential confounds linked to population stratification; (2) results from SFEs are weighted more heavily in college admissions and retaking the examination is more difficult and occurs less frequently than similar tests in the US, and (3) examinations occurred on the same days for all students which made data collection more practical as all students could be tested in large numbers at the same location.

\section{Procedure}

During both sessions, participants completed a probabilistic reward learning task $[8,26]$. During session 1 , participants filled out a demographics and handedness questionnaire [28]. In addi- tion, the following self-report measures were administered at each session to assess depression, hedonic capacity, anxiety, and perceived stress: (1) the Beck Depression Inventory II (BDI II [29]); (2) the Mood and Anxiety Symptom Questionnaire [30]; (3) the Perceived Stress Scale [31]; (4) a questionnaire assessing schooland examination-related stress, specifically developed for the study (available upon request); (5) drug use questionnaire assessing lifetime and recent (previous month) use of common substances (available upon request); (6) a self-report measure of family or personal history of depression or bipolar disorder, modified after Berenbaum and Connelly [7], and (7) the Temporal Experience of Pleasure Scale (TEPS) [32] (for exploratory purposes, not included in current analyses). All questionnaires were translated into Bulgarian by the first author and translated back into English by a different Bulgarian native to ensure the integrity of translation. At session 1, all participants provided saliva samples for DNA analysis (Oragene, DNA Genotek; Ottawa, Ont., Canada).

\section{Probabilistic Reward Task}

A probabilistic reward task was used to measure reward responsiveness $[10,33]$. The task consisted of 3 blocks of 80 trials. In each trial, participants were instructed to identify whether a mouth presented briefly (i.e. for $100 \mathrm{~ms}$ ) on a schematic face was either long or short (long mouth: $11.00 \mathrm{~mm}$, short mouth: 10.00 $\mathrm{mm}$ ) by making an appropriate response on a computer keyboard (using the ' $f$ ' or ' $j$ ' keys; counterbalanced). The main dependent variables were response bias (a participant's propensity to select one stimulus regardless of actual stimulus presentation) and discriminability (a participant's ability to perceptually distinguish the two stimuli). Importantly, the small size difference between stimuli, as well as the short duration of stimulus exposure, made it difficult to ascertain which stimulus was presented and an asymmetric reward schedule was used to induce a response bias $[10,33]$. Specifically, in each block, correct identification of either the short or the long stimulus ('rich stimulus') was rewarded ('Correct!! You won 0.15 Leva') three times more frequently than correct identification of the other stimulus ('lean stimulus'). Prior to the experiment, participants were informed that the goal of the study was to win as much money as possible and that not all correct responses would be rewarded. No information was provided regarding the differential reinforcement schedule.

To ensure similar reward ratio exposure across subjects, only 32 correct trials ( 24 rich, 8 lean) were scheduled to receive reward according to a controlled reinforcement procedure [10]; thus, if a participant was incorrect on a trial scheduled to receive reward, reward feedback was delayed until the next correct identification of the same stimulus type. The stimulus list and reinforcement sequence were pseudorandomized with the constraint that no stimulus could be presented more than 3 times in a row and that each block ended with 3 unscheduled rewards for each stimulus type (to increase the likelihood that participants would receive all planned rewards). For each participant, all task parameters were kept unchanged across the two sessions.

Importantly, in community, clinical, and student samples reward responsiveness as measured by this task has been found to be: (1) blunted in participants with depression [34]; (2) blunted under an acute laboratory stressor [8, 35]; (3) heritable and genetically associated with perceived stress [36], and (4) influenced by the interaction of stress-related genotypes and stress [9]. 


\section{Data Reduction and Processing}

Probabilistic Reward Task. Prior to analyses, outlier responses were identified with a two-step procedure; first, trials with reaction times (RTs) shorter than $150 \mathrm{~ms}$ or longer than $1,500 \mathrm{~ms}$ were excluded; second, after applying a natural log transformation to normalize RT distribution, trials with mean \pm 3 SD were excluded for each participant and participants with more than 30 (i.e. $12.5 \%)$ outlier trials were excluded from the analyses. Following outlier removal, response bias $(\log b)$ and discriminability $(\log d)$ were calculated as follows [11]:

Response bias: $\log b=\frac{1}{2} \log \left(\frac{\left(\text { Rich }_{\text {correct }}+0.05\right) \cdot\left(\text { Lean }_{\text {incorrect }}+0.05\right)}{\left(\text { Rich }_{\text {incorrect }}+0.05\right) \cdot\left(\text { Lean }_{\text {correct }}+0.05\right)}\right)$

Discriminability: $\log d=\frac{1}{2} \log \left(\frac{\left(\text { Rich }_{\text {correct }}+0.05\right) \cdot\left(\text { Lean }_{\text {correct }}+0.05\right)}{\left(\text { Rich }_{\text {incorrect }}+0.05\right) \cdot\left(\text { Lean }_{\text {incorrect }}+0.05\right)}\right)$.

A high response bias emerges when participants rarely miss presentation of stimulus rewarded more frequently (rich stimulus) and tend to misclassify the lean stimulus as the rich stimulus.

Exam-Related Stress. A perceived SFE stress subscale was computed by summing four items from the questionnaire assessing school and examination-related stress developed specifically for this study. Two of the items were ranked on a 1-7 Likert Scale ('How worried are you about your SFEs?' and 'How prepared are you for your SFEs?'). The remaining two items asked participants to provide an estimate of the grade they expected to receive on each of their two compulsory SFEs. These grade estimates were subsequently converted to a 1-7 scale, with higher stress scores indicating lower grade estimates. ${ }^{1}$ After reverse-coding the item probing examination preparedness, the four items were summed, so that higher scores indicated higher levels of SFE-related stress (possible range: 4-28; actual range in the current sample: 6-25). The SFE stress subscale had satisfactory internal consistency across the two sessions (Cronbach's $\alpha=0.68$ ), especially in light of the small number of items [37].

Initially, session 1 was designed to be the control condition, and session 2 the stress condition. However, self-report measure of SFE stress unexpectedly indicated that the majority of students experienced less stress at session 2 , immediately prior to their examinations, relative to session 1. [This finding may be explained by the reduced uncertainty regarding the SFE format and better examination preparation received over the course of the second half of the school year. Additionally, students may have experienced more stress during session 1 , due to the fact that the beginning of the school year was delayed by 6 weeks because of a nationwide teachers' strike. To corroborate this assumption, participants in session 1 were asked to answer the following question 'Did the fact that the school year started later due to the teachers' strike make you more or less stressed about school, relative to previous school years?' using a 7-point scale (1: completely relaxed, 7: extremely stressed). A one-sample $t$ test revealed that participants' score $(4.56 \pm 1.24)$ significantly deviated from the midpoint, highlighting enhanced levels of stress, $\mathrm{t}(68)=3.78, \mathrm{p}<$

1 In Bulgaria, examinations are graded on a 2.00-6.00 point scale, 3.00 being the lowest passing grade. Thus, the expected grades were converted into stress scores as follows: $<3=$ add $7,3-3.49=$ add $6,3.5-3.99=$ add 5 , $4-4.49=$ add $4,4.5-4.99=$ add $3,5-5.49=$ add $2, \geq 5.5=$ add 1 .
0.001; one student did not provide a score and could thus not be included in this analysis.]

Based on this additional information, participants' data were regrouped into stress and control conditions based on individual self-report measures of exam-related stress in each session. Specifically, the session in which each participant reported higher SFE stress was considered his/her stress condition. Accordingly, session 1 was the stress condition for 44 participants, whereas session 2 was the stress condition for 16 participants. There were no differences in age, gender or genotype between these groups (all p >0.27). Ten participants had identical levels of SFE stress in both sessions and were not included in analyses involving within-subject comparisons. Per design, stress scores were higher in the stress $(14.50 \pm$ 3.98) than in the control $(11.52 \pm 3.23)$ condition $[\mathrm{t}(60)=11.34$, $\mathrm{p}<0.001]$. Importantly, even though session 1 was associated with more SFE stress overall, it was no more likely to be designated as the stress condition for one gender or genotype group than another (both $\chi^{2}<3.56$ and $p>0.10$ ), thus precluding the possibility that order or practice effects may have influenced our results.

Genotyping. 5-HTTLPR and SNP (rs25531) genotyping was performed according to established procedures [38]. In $20 \mu \mathrm{l}$, Genomic DNA (25 ng) was amplified via a polymerase chain reaction in the presence of $1 \mathrm{x}$ multiplex master mix (Qiagen, Valencia, Calif., USA) and primers (forward: 5'-TCCTCCGCTTTGGCGCCTCTTCC-3'; reverse: 5'-TGGGGGTTGCAGGGGAGATCCTG-3'; Integrated DNA Technologies, Coralvillem, Iowa, USA). Next, $7 \mu 1$ of PCR product was digested by HpaII (13 $\mu \mathrm{l}$; New England BioLabs, Ipswich, Mass., USA) in a reaction assay with $1 \times$ NEBuffer1 and $1 \times$ BSA (Ambion, Foster City, Calif., USA). Finally, $4 \mu \mathrm{l}$ of the remaining PCR product and $18 \mu \mathrm{l}$ of restriction enzyme assay solution were loaded onto a 2.0\% agarose gel (E-Gel, Invitrogen, Carlsbad, Calif., USA) and were visualized after 15, 25 and 45 min. Participants carrying at least one copy of the low expressing $\mathrm{S}$ or $\mathrm{L}_{\mathrm{G}}$ alleles were grouped together and are collectively referred to as ' $S$ ' participants' throughout the analyses. Participants homozygous for the high expressing $\mathrm{L}_{\mathrm{A}}$ allele are referred to as ' $\mathrm{L}$ ' participants'.

\section{Statistical Analyses}

Two sets of analyses were conducted. In the first set, a series of genotype $\times$ condition ANOVAs were conducted on several selfreport measures to evaluate whether genotype groups differed in their report of mood; these analyses were considered secondary and were performed to ensure that our primary findings (group differences in reward responsiveness) were not unduly affected by differences in self-report measures of mood. Next, we evaluated the possible moderating effect of 5-HTTLPR/rs25531 genotype on task performance, which represented the main analysis of interest. After rebinning the data into stress and control conditions, a mixed ANOVA with 5-HTTLPR/rs25531 genotype (S' participants, L' participants) as between-subject factor and block $(1,2,3)$ and condition (stress, control) as within-subject factors was conducted on a response bias. Control analyses were performed by entering gender and change in depressive symptoms between the two conditions as covariates in an ANCOVA. These control analyses were performed in light of reports showing (1) gender differences in depression following stress [39] and the association between 5-HTTLPR, stress and depression [25, 26, 40], and (2) decreased response bias in depression [10, 41]. Accordingly, the analyses probed putative genotype $\times$ condition effects of perceived SFE stress independent of gender and general dysphoria or 
Table 1. Summary of control analyses assessing self-report measures of mood and affect as a function of condition and genotype: no main effects of genotype or condition $\times$ genotype interactions emerged for any of these measures, thus emphasizing that our findings are not due to differences in perceived stress between S' participants and L' participants

\begin{tabular}{|c|c|c|c|c|c|c|}
\hline & \multicolumn{2}{|l|}{ Control } & \multicolumn{2}{|l|}{ Stress } & \multirow[t]{2}{*}{ Effect } & \multirow[t]{2}{*}{$\mathrm{p}$ values } \\
\hline & $\begin{array}{l}\text { S' participants } \\
(\mathrm{n}=44)\end{array}$ & $\begin{array}{l}\text { L' participants } \\
(\mathrm{n}=16)\end{array}$ & $\begin{array}{l}\text { S' participants } \\
(\mathrm{n}=44)\end{array}$ & $\begin{array}{l}\text { L' participants } \\
(\mathrm{n}=16)\end{array}$ & & \\
\hline SFE stress & $11.50 \pm 2.90$ & $11.56 \pm 4.13$ & $14.61 \pm 3.87$ & $14.19 \pm 4.40$ & condition & $<0.001$ \\
\hline PSS & $28.82 \pm 7.48$ & $27.00 \pm 8.51$ & $30.48 \pm 7.77$ & $31.44 \pm 9.41$ & condition & 0.016 \\
\hline BDI-II & $14.07 \pm 9.59$ & $13.13 \pm 11.40$ & $14.60 \pm 9.22$ & $15.38 \pm 11.87$ & none & $>0.27$ \\
\hline \multicolumn{7}{|l|}{ MASQ } \\
\hline GDA & $21.81 \pm 8.37$ & $18.19 \pm 6.72$ & $21.11 \pm 7.46$ & $21.31 \pm 7.93$ & none & $>0.53$ \\
\hline $\mathrm{AA}$ & $29.71 \pm 11.90$ & $26.50 \pm 6.38$ & $31.12 \pm 12.59$ & $28.06 \pm 10.86$ & none & $>0.26$ \\
\hline GDD & $22.62 \pm 9.83$ & $20.81 \pm 12.38$ & $23.52 \pm 9.61$ & $25.13 \pm 11.77$ & condition & 0.041 \\
\hline $\mathrm{AD}$ & $56.65 \pm 15.41$ & $51.41 \pm 17.46$ & $58.22 \pm 9.82$ & $60.81 \pm 17.03$ & condition & 0.027 \\
\hline
\end{tabular}

SFE = School final examinations; PSS = Perceived Stress Scale; BDI-II = Beck Depression Inventory II; MASQ = Mood and Anxiety Symptom Questionnaire; GDA = general distress anxiety; AA = anxious arousal; GDD = general distress depression; $\mathrm{AD}=$ anhedonic depression.

depressive symptoms, which was particularly important given the rebinning of the stress and control conditions. To assess depressive symptoms, the MASQ GDD subscale was preferred over the $\mathrm{BDI}$, because it provides a measure of general depression score, independent of anhedonic symptoms.

In a second set of analyses, to directly investigate the role of gender on task performance, a genotype $\times$ block $\times$ condition $\times$ gender ANCOVA (controlling for GDD score change between conditions) was performed on response bias and discriminability.

\section{Results}

\section{Demographics and Self-Report Data}

A series of genotype (S' participants, L' participants) $\times$ condition (stress, control) ANOVAs revealed that MASQ GDD, MASQ AD, and PSS scores were higher in the stress relative to control condition (table 1), providing further corroboration for the data rebinning strategy based on SFE stress scores. Importantly, all other effects, including the genotype $\times$ condition interaction, were not significant (all p values $>0.11$ ), indicating that genotype groups did not differ with respect to other self-report measures of mood.

\section{Task Performance}

The 5-HTTLPR/rs25531 genotype $\times$ block $\times$ condition ANOVA for response bias revealed a main effect of block $\left[F(2,116)=6.95\right.$, $p<0.002$, partial $\left.\eta^{2}=0.107\right]$, due to heightened response bias in blocks $3(0.197 \pm 0.019)$ and $2(0.170 \pm 0.018)$ relative to block $1(0.127 \pm 0.017)$ (both $\mathrm{p}<0.02$ ). This finding confirms that the task induced the expected preference for the more frequently rewarded stimulus, which increased as the task progressed.

Most importantly, a significant genotype $\times$ condition interaction emerged, $[\mathrm{F}(1,58)=4.38, \mathrm{p}<0.05$, partial $\eta^{2}=0.070$; fig. 1a], due to significantly larger stress-related reduction in response bias in S' participants than L' participants (response bias ${ }_{\text {Control }}$ - response bias ${ }_{S t r e s s}$ : $0.035 \pm 0.150$ vs. $-0.061 \pm 0.128$; Cohen's $d=0.67)^{2}$. Critically, the group difference in stress-related reduction in response bias remained significant after controlling for gender and GDD change between conditions $[F(1,55)=$ 5.83, $\mathrm{p}<0.02$, partial $\eta^{2}=0.096$ ]. Independent-samples $t$ tests revealed no differences between genotypes in either the stress or the control condition (both $\mathrm{p}>0.15$ ), indicating that genotype groups differed significantly only in their relative stress-related change in response bias.

\footnotetext{
2 In a previous study, we observed an effect size (Cohen's d) of 0.89 for differences in reward responsiveness under an acute stress manipulation between groups differing in genetic variants within the mineralocorticoid receptor (MR) gene [9]. Similar to the 5-HTTLPR/rs25531 genotype, the MR gene has been implicated in depression and modulating stress responses (e.g. [60-62]). Power analyses indicate that two groups of 45 and 15 subjects, respectively, were needed to detect an effect size of 0.89 with a power of 0.90 . Thus, the current sample of 44 S' carriers and 16 L' homozygotes provided enough power to detect similar effects.
} 


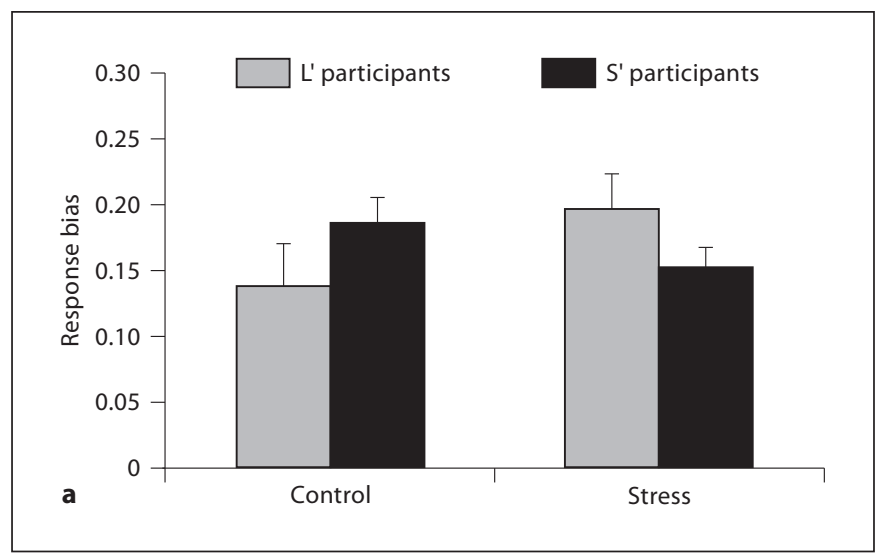

Fig. 1. Effects of condition and 5-HTTLPR/rs25531 genotype on response bias. There was a significant genotype $\times$ condition interaction, whereby the two genotype groups differed in their relative reduction in response bias from the control to the stress condition $\left[\mathrm{F}(1,58)=4.38, \mathrm{p}<0.05\right.$, partial $\left.\eta^{2}=0.070\right]$. S' participants ( $\mathrm{n}=44$; black bars) showed a relative decrease from the control $(0.186 \pm 0.137)$ to the stress $(0.150 \pm 0.117)$ condition, while L' participants ( $\mathrm{n}=16$; gray bars) exhibited a relative increase (control: $0.129 \pm 0.121$; stress: $0.191 \pm 0.087)(\mathbf{a})$. A genotype $\times$ condition $\times$ gender interaction indicated that the effect was present in males (S' participants: $n=24$, L' participants: $n=9)(\mathbf{b})$, but not females (S' participants: $n=20$, L' participants: $n=7$ ) (c). Error bars represent the SEM.

To examine the effects of gender, a genotype $\times$ block $\times$ condition $\times$ gender ANCOVA was conducted on response bias, while controlling for GDD change between conditions. In addition to the genotype $\times$ condition interaction $\left[\mathrm{F}(1,54)=4.92, \mathrm{p}<0.04\right.$, partial $\left.\eta^{2}=0.084\right]$, a significant three-way genotype $\times$ condition $\times$ gender interaction emerged $\left[\mathrm{F}(1,54)=4.43, \mathrm{p}<0.05\right.$, partial $\eta^{2}=$ 0.076]. Highlighting the specificity of these findings, a control genotype $\times$ block $\times$ condition $\times$ gender ANCOVA on discriminability revealed no significant effects, all $\mathrm{p}>0.15$.

When genotype $\times$ block $\times$ condition ANCOVAs were conducted on response bias separately for males and females, a significant genotype $\times$ condition interaction was observed in males $[\mathrm{F}(1,26)=14.15, \mathrm{p}<0.002$, partial $\eta^{2}=0.352$; fig. $\left.1 b\right]$, but not females (all $p>0.50$; fig. 1c). Follow-up within-subject $t$ tests showed that male S' participants were characterized by a significant reduction in total response bias from the control $(0.218 \pm 0.127)$ to the stress $(0.147 \pm 0.120)$ condition $[t(19)=-2.26, \mathrm{p}<0.04]$, while male L' participants showed a significant increase
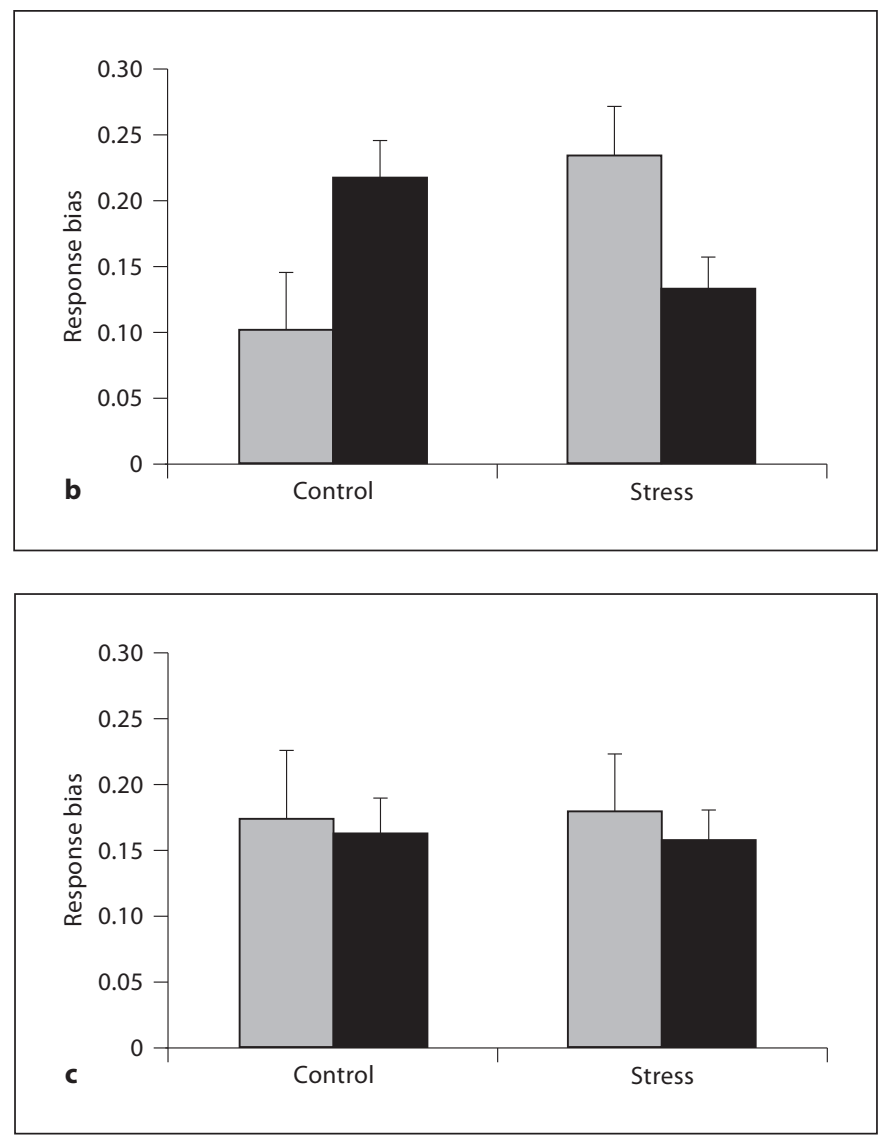

[control condition: $0.093 \pm 0.115$, stress condition: 0.197 $\pm 0.098 ; \mathrm{t}(8)=2.68, \mathrm{p}<0.03$; fig. $1 \mathrm{~b}]$. When GDD change was controlled for, the increase in response bias in L' participants was reduced to a trend $[\mathrm{F}(1,7)=4.34$, $p=0.076$, partial $\eta^{2}=0.383$, while the decrease in response bias in S' participants remained significant $\left[\mathrm{F}(1,18)=7.24, \mathrm{p}<0.02\right.$, partial $\left.\eta^{2}=0.287\right]$.

\section{Discussion}

The main goal of the present study was to examine how perceived stress associated with a naturalistic stressor (school examinations) and 5-HTTLPR/rs25531 genotype jointly influence reward responsiveness, a core component of anhedonia. The main finding emerging from this study was that 5-HTTLPR/rs25531 genotype modulated susceptibility to stress-related reward responsiveness impairments in males; specifically male S' participants were characterized by significantly larger stress-related reduction in response bias relative to L' 
participants (significant genotype $\times$ condition interaction). Notably, the interaction was due to lower response bias in the stress relative to control condition for S' participants, whereas L' participants showed the opposite pattern. Critically, stress-related response bias modulations emerged within the context of no differences in discriminability, indicating that the effects of stress were specific to the ability to modulate behavior as a function of reinforcement history. In light of the fact that anhedonia is a core symptom of depression and stress-related hedonic deficits have been hypothesized to underlie the depressogenic effects of stress $[5,8]$, our findings suggest potential environmental and genetic etiological mechanisms that may influence the development of depression. To the best of our knowledge, this is the first study to demonstrate an interactive effect of 5-HTTLPR/rs25531 genotype and stress on reward processing in humans.

\section{5-HTTLPR/rs25531 Genotype, Stress and Reward Processing}

The stress-mediated reduction in reward learning we observed in male S' participants could be conceptualized as the result of several distinct, but not mutually exclusive, phenomena. In one of the earliest studies investigating the potential involvement of stress-related anhedonia in humans, Berenbaum and Connelly [7] found that stress reduced the subjective hedonic value of activities normally experienced as enjoyable. The blunted reward responsiveness we observed could be interpreted as the consequence of similar reductions in the subjective pleasure derived from earning a monetary reward in the experimental task (or simply doing well in it) - an activity normally associated with enjoyment. Additionally, since stress is apt to induce large shifts in behavioral priorities [42], our findings could also reflect stress-related reduction in the incentive value assigned to positive stimuli, which may in turn reflect a more global change in motivational state geared towards effectively dealing with a stressor. On the neurobiological level, and in line with the modulatory role of 5-HT on dopaminergic transmission, the reward processing deficits we observed in male S' participants could be attributed to stress-mediated decrease in dopamine signaling in neural regions critically implicated in reinforcement learning and motivation $[19,43$, 44]. Neuroimaging techniques such as multimodal PET/ fMRI will be required to test this conjecture and clarify the precise mechanism via which stress and 5-HT signaling modulate dopaminergic transmission and reward processing.

Stress Perception, 5-HTTLPR Genotype and Reward
Although we had not predicted the relative stress-related increase in reward responsiveness in L' participants, our results are in line with the proposition that the association among 5-HTTLPR, stress, and depression could at least partially be explained by conceptualizing the L' genotype as conferring emotional resiliency in the face of stress and trauma $[45,46]$. The increased reward responsiveness in L' participants under stress in our sample could thus be interpreted as a potential mechanism via which the L' allele exerts its putative protective effects. Such an interpretation is consistent with the notion that a robust reward system is an essential component of resilience $[47,48]$, and is further corroborated by research demonstrating that L' homozygotes show positive information processing biases absent in S' carriers $[49,50]$.

The current results are also in line with findings from the animal literature. A recent study reported that priming with a social stressor (photographs of high-ranking males) led to heightened risk aversion in rhesus macaque monkeys carrying one copy of the S allele of the rh5HTTLPR (homologous to the human 5-HTTLPR), but increased risk-seeking in individuals homozygous for the L allele, which the authors conceptualized as an enhanced sensitivity to social punishment and reward, respectively [51]. Our results are also consistent with reports from rodent studies suggesting that chronic mild stress can in certain cases enhance sensitivity to pleasurable stimuli (i.e. causing 'prohedonia'), rather than decrease it [6]. Critically, whether chronic mild stress results in anhedonia or prohedonia depends on a variety of variables, including the genetic makeup of the animal under study $[52,53]$.

\section{Role of Gender}

While prior research has suggested that the association between the 5-HTTLPR S allele, stress, and depression may be specific to females [54], we found no association between genotype and stress-related response bias changes in females. These findings might be accounted for by the type of the stressor we employed. Depressive symptoms and biological stress responses have been associated with different types of stressful life events in a gender-specific manner, with females and males being most sensitive to interpersonal stressors and achievement stressors, respectively [55]. Because the naturalistic stressor we investigated was an important school examination, the males in our sample may have been disproportionately affected by it. In addition, the male-specific nature of our findings may be related to the fact that the reward we used in our task was monetary, and males have 
been shown to respond more strongly to monetary reward feedback than females [56]. The gender effects in the current study must, however, be interpreted with caution due to the small sample size of these analyses.

\section{Limitations and Future Directions}

The present study has several limitations. First, unexpectedly, session 1 and session 2 were not associated with the intended levels of stress and thus did not map directly onto our control and stress conditions, respectively. We believe this unexpected pattern was due to extraneous factors that led to a greater uncertainty surrounding the SFEs in December (e.g. 6-week, nationwide teachers' strike, first time these exams had been introduced in Bulgaria), rather than May, increasing stress levels at the originally planned control condition. In order to address this problem, we re-grouped data into control and stress conditions based on self-report measures of SFE-related stress. Though not directly tapping into the physiological correlates of stress, our scale can be considered a valid measure of exam-related stress, as classic research has shown that perceived importance of a particular goal, combined with a perceived low probability of attaining that goal, is associated with significant distress [57]. These classic findings, together with the significant differences in self-reported SFE stress and PSS after the rebinning, lend credibility to our approach. Of note, this regrouping had the additional advantage that, unlike in the original design, order effects were minimized due to a partial counterbalancing across subjects. However, the fact that many participants reported higher levels of stress at time 1 compared to time 2 prevents us from conclusively stating that the response bias findings were driven by stress associated with the final examinations. Nevertheless, the lack of genotype differences in (1) self-report of mood, including SFE-related stress, at both sessions, and (2) the percentage of participants reporting higher levels of stress at time 1 than time 2 , as well as the fact that SFE scores were used to define the stress and control condition for each participants, lend support to our interpretation that exam-related perceived stress contributed to the reported gene $\times$ condition effects on reward responsiveness.

Second, our study focused on perceived stress (i.e. on the subjective impact of events), rather than more objective indices (e.g. interviewer-assessed levels of contextual threat) or physiological markers (e.g. cortisol). It has been argued that perceived stress can be confounded with depressive symptoms, which might inflate links between the independent and dependent variable (e.g. [58]). To ad- dress this legitimate criticism, we performed control analyses adjusting for depressive symptoms (MASQ GDD scores), which confirmed the finding of stress-related reduction in response bias among S' participants. Moreover, as mentioned above, genotype groups did not differ in any self-report measures of mood, including SFE-related stress scores, indicating that 5-HTTLPR/ rs25531 genotype did not impact perceptions of stress.

Third, due to the relatively small sample size of this study, our results should be interpreted with caution until replicated in larger samples. This precaution is particularly important in relation to the three-way condition $\times$ genotype $\times$ gender interaction. We note, however, that the current findings in S' carriers are consistent with the results from three prior independent studies highlighting reduced reward responsiveness, as assessed by our probabilistic reward task, under stressful situations $[8,9,59]$. Accordingly, replication of stress-induced reduction in reward responsiveness has now been obtained in four independent samples, strengthening our confidence that the current findings are reliable. Finally, while we excluded participants reporting drug use and/or a personal or familial history of MDD or bipolar disorder based on self-report, and collected well-validated measures of depressive and anxious symptomatology, we did not formally assess all Axis I or II disorders with structured clinical interviews.

Limitations notwithstanding, the current investigation extends previous work suggesting that the depressogenic effect of stress may be at least partially driven by the emergence of a reduced ability to modulate behavior based on prior reinforcement under stressful situations $[7,8]$. Furthermore, our findings highlight stress-related reward responsiveness deficits as a promising mechanism underlying the association between 5-HTTLPR genotype, stress, and depression. Additionally, the 'prohedonic' behavior that L' participants exhibited under stress may be interpreted as protective against MDD development in the face of life adversity. To further elucidate this association, future studies should utilize larger samples to examine the behavioral and neural correlates of reward processing in relation to objective measures of stress and 5-HTTLPR/rs25531 genotype, while taking into account the severity and type of the stressor, as well as any relevant gene-gene interactions. 


\section{Acknowledgements}

Funding for this study was provided by grants from the Harvard College Research Program to Y.N., grant from NIMH (R01MH68376, R21MH078979) and Talley Fund (Harvard University) to D.A.P., and a Sackler Scholar in Psychobiology Research Grant to R.B. The authors are grateful to Ahmad Hariri for providing genotyping information, to Allison Brown
(Harvard Medical School-Partners Healthcare Center for Genetics and Genomics), Sara Dainese and Emily Yuan for their assistance in the genotyping process, and to Vesselin Dimitrov for translating study material.

Diego A. Pizzagalli has received research support from GlaxoSmithKline, consulting fees from ANT North America Inc. (Advanced Neuro Technology) and AstraZeneca, and honoraria from AstraZeneca for projects unrelated to the present study.

\section{References}

1 Brown GW HT: Social Origins of Depression: A Study of Psychiatric Disorder in Women. New York, Free Press, 1978.

- 2 Kendler KS, Karkowski LM, Prescott CA: Causal relationship between stressful life events and the onset of major depression. Am J Psychiatry 1999;156:837-841.

-3 Paykel ES, Myers JK, Dienelt MN, Klerman GL, Lindenthal JJ, Pepper MP: Life events and depression: a controlled study. Arch Gen Psychiatry 1969;21:753-760.

4 van Praag HM: Can stress cause depression? Prog Neuro-Psychoph 2004;28:891-907.

5 Anisman H, Matheson K: Stress, depression, and anhedonia: caveats concerning animal models. Neurosci Biobehav Rev 2005;29: 525-546.

6 Willner P: Chronic mild stress (CMS) revisited: consistency and behavioural-neurobiological concordance in the effects of CMS. Neuropsychobiology 2005;52:90-110.

7 Berenbaum H, Connelly J: The effect of stress on hedonic capacity. J Abnorm Psychol 1993; 102:474-481.

-8 Bogdan R, Pizzagalli DA: Acute stress reduces reward responsiveness: Implications for depression. Biol Psychiatry 2006;60:11471154.

-9 Bogdan R, Perlis RH, Fagerness J, Pizzagalli DA: The impact of mineralocorticoid receptor iso/val genotype (rs5522) and stress on reward learning. Genes Brain Behav 2010;9: 658-667.

10 Pizzagalli DA, Jahn AL, O’Shea JP: Toward an objective characterization of an anhedonic phenotype: a signal-detection approach. Biol Psychiatry 2005;57:319-327.

- 11 Pizzagalli DA, Bogdan R, Ratner KG, Jahn AL: Increased perceived stress is associated with blunted hedonic capacity: potential implications for depression research. Behav Res Ther 2007;45:2742-2753.

12 Pruessner JC, Dedovic K, Khalili-Mahani N, Engert V, Pruessner M, Buss C, Renwick R, Dagher A, Meaney MJ, Lupien S: Deactivation of the limbic system during acute psychosocial stress: evidence from positron emission tomography and functional magnetic resonance imaging studies. Biol Psychiatry 2008;63:234-240.
13 Lumley MN, Harkness, KL: Specificity in the relations among childhood adversity, early maladaptive schemas, and symptom profiles in adolescent depression. Cogn Ther Res 2007;31:639-657.

14 Dillon DG, Holmes AJ, Birk JL, Brooks N, Lyons-Ruth K, Pizzagalli DA: Childhood adversity is associated with left basal ganglia dysfunction during reward anticipation in adulthood. Biol Psychiatry 2009;66:206-213.

15 Bruijnzeel AW: Kappa-opioid receptor signaling and brain reward function. Brain Res Rev 2009;62:127-146.

16 Bearer EL, Zhang X, Janvelyan D, Boulat B, Jacobs RE: Reward circuitry is perturbed in the absence of the serotonin transporter. NeuroImage 2009;46:1091-1104.

17 Sanders AC, Hussain AJ, Hen R, Zhuang X: Chronic blockade or constitutive deletion of the serotonin transporter reduces operant responding for food reward. Neuropsychopharmacology 2007;32:2321-2329.

18 Dremencov E, El Mansari M, Blier P: Effects of sustained serotonin reuptake inhibition on the firing of dopamine neurons in the rat ventral tegmental area. J Psychiatry Neurosci 2009;34:223-229.

19 Schultz W: Getting formal with dopamine and reward. Neuron 2002;36:241-263.

20 Heils A, Teufel A, Petri S, Stober G, Riederer P, Bengel D, Lesch KP: Allelic variation of human serotonin transporter gene expression. J Neurochem 1996;66:2621-2624.

21 Caspi A, Sugden K, Moffitt TE, Taylor A, Craig IW, Harrington H: Influence of life stress on depression: moderation by a polymorphism in the 5-htt gene. Science 2003; 301:386-389.

22 Risch N, Herrell R, Lehner T, Liang KY, Eaves L, Hoh J, Griem A, Kovacs M, Ott J, Merikangas KR: Interaction between the serotonin transporter gene (5-HTTLPR), stressful life events, and risk of depression: a meta-analysis. JAMA 2009;301:2462-2471.

23 Hu X, Oroszi G, Chun J, Smith TL, Goldman D, Schuckit MA: An expanded evaluation of the relationship of four alleles to the level of response to alcohol and the alcoholism risk. Alcohol Clin Exp Res 2005;29:8-16.
24 Zalsman G, Huang YY, Oquendo MA, Burke AK, Hu XZ, Brent DA, Ellis SP, Goldman D, Mann JJ: Association of a triallelic serotonin transporter gene promoter region (5HTTLPR) polymorphism with stressful life events and severity of depression. Am J Psychiatry 2006;163:1588-1593.

25 Brummett BH, Boyle SH, Siegler IC, Kuhn CM, Ashley-Koch A, Jonassaint CR, Zuchner S, Collins A, Williams RB: Effects of environmental stress and gender on associations among symptoms of depression and the serotonin transporter gene linked polymorphic region (5-HTTLPR). Behav Genet 2008;38:34-43.

26 Sjoberg RL, Nilsson KW, Nordquist N, Ohrvik J, Leppert J, Lindstrom L, Oreland L: Development of depression: sex and the interaction between environment and a promoter polymorphism of the serotonin transporter gene. Int J Neuropsychopharmacol 2006;9:443-449.

27 Dickerson SS, Kemeny ME: Acute stressors and cortisol responses: a theoretical integration and synthesis of laboratory research. Psychol Bull 2004;130:355-391.

28 Chapman LJ, Chapman JP: The measurement of handedness. Brain Cogn 1987;6: 175-183.

29 Beck A, Steer RA, Brown GK: Beck Depression Inventory Manual, ed 2. San Antonio, The Psychological Corporation, 1996.

30 Watson D, Weber K, Assenheimer JS, Clark LA, Strauss ME, Mccormick RA: Testing a tripartite model. I. Evaluating the convergent and discriminant validity of anxiety and depression symptom scales. J Abnorm Psychol 1995;104:3-14.

31 Cohen S, Kamarck T, Mermelstein R: A global measure of perceived stress. J Health Soc Behav 1983;24:385-396.

32 Gard DE, Gard MG, Kring AM, John OP: Anticipatory and consummatory components of the experience of pleasure: a scale development study. J Res Pers 2006;40:10861102.

33 Tripp G, Alsop B: Sensitivity to reward frequency in boys with attention deficit hyperactivity disorder. J Clin Child Psychol 1999; 28:366-375. 
-34 Pizzagalli DA, Iosifescu D, Hallett LA, Ratner KG, Fava M: Reduced hedonic capacity in major depressive disorder: evidence from a probabilistic reward task. J Psychiatr Res 2009;43:76-87.

-35 Liu WH, Chan RC, Wang LZ, Huang J, Cheung EF, Gong QY, Gollan JK: Deficits in sustaining reward responses in subsyndromal and syndromal major depression. Prog Neuropsychopharmacol Biol Psychiatry 2011;35:1045-1052.

-36 Bogdan R, Pizzagalli DA: The heritability of hedonic capacity and perceived stress: a twin study evaluation of candidate depressive phenotypes. Psychol Med 2009;39:211-218.

$\checkmark 37$ Moss S, Prosser H, Costello H, Simpson N, Patel P, Rowe S, Turner S, Hatton C: Reliability and validity of the pas-add checklist for detecting psychiatric disorders in adults with intellectual disability. J Intellect Disabil Res 1998;42:173-183.

38 Wendland JR, Martin BJ, Kruse MR, Lesch KP, Murphy DL: Simultaneous genotyping of four functional loci of human slc6a4, with a reappraisal of 5-HTTLPR and rs25531. Mol Psychiatry 2006;11:224-226.

39 Kendler KS, Kuhn J, Prescott CA: The interrelationship of neuroticism, sex, and stressful life events in the prediction of episodes of major depression. Am J Psychiatry 2004;161: 631-636.

-40 Mandelli L, Serretti A, Marino E, Pirovano A, Calati R, Colombo C: Interaction between serotonin transporter gene, catechol-omethyltransferase gene and stressful life events in mood disorders. Int J Neuropsychopharmacol 2007; 10:437-447.

-41 Pizzagalli DA, Holmes AJ, Dillon DG, Goetz EL, Birk JL, Bogdan R, Dougherty DD, Iosifescu DV, Rauch SL, Fava M: Reduced caudate and nucleus accumbens response to rewards in unmedicated individuals with major depressive disorder. Am J Psychiatry 2009; 166:702-710

-42 Johnson EO, Kamilaris TC, Chrousos GP, Gold PW: Mechanisms of stress: a dynamic overview of hormonal and behavioral homeostasis. Neurosci Biobehav Rev 1992;16: 115-130.
43 Cabib S, Puglisi-Allegra S: Stress, depression and the mesolimbic dopamine system. Psychopharmacology (Berl) 1996;128:331-342.

44 Mangiavacchi S, Masi F, Scheggi S, Leggio B, De Montis MG, Gambarana C: Long-term behavioral and neurochemical effects of chronic stress exposure in rats. J Neurochem 2001;79:1113-1121.

45 Kohen R, Cain KC, Mitchell PH, Becker K, Buzaitis A, Millard SP, Navaja GP, Teri L, Tirschwell D, Veith R: Association of serotonin transporter gene polymorphisms with poststroke depression. Arch Gen Psychiatry 2008;65:1296-1302.

46 Stein MB, Campbell-Sills L, Gelernter J: Genetic variation in 5HTTLPR is associated with emotional resilience. Am J Med Genet B Neuropsychiatr Genet 2009;150B:900906.

47 Charney DS: Psychobiological mechanisms of resilience and vulnerability: implications for successful adaptation to extreme stress. Am J Psychiatry 2004;161:195-216.

48 Feder A, Nestler EJ, Charney DS: Psychobiology and molecular genetics of resilience. Nat Rev Neurosci 2009; 10:446-457.

49 Fox E, Ridgewell A, Ashwin C: Looking on the bright side: biased attention and the human serotonin transporter gene. Proc Biol Sci 2009;276:1747-1751.

50 Perez-Edgar K, Bar-Haim Y, McDermott JM, Gorodetsky E, Hodgkinson CA, Goldman D, Ernst M, Pine DS, Fox NA: Variations in the serotonin-transporter gene are associated with attention bias patterns to positive and negative emotion faces. Biol Psychol 2010;83: 269-271.

51 Watson KK, Ghodasra JH, Platt ML: Serotonin transporter genotype modulates social reward and punishment in rhesus macaques. PLoS One 2009;4:e4156.

52 Martin M, Ledent C, Parmentier M, Maldonado R, Valverde O: Involvement of cb1 cannabinoid receptors in emotional behaviour. Psychopharmacology (Berl) 2002;159:379_ 387.

53 Nielsen CK, Arnt J, Sanchez C: Intracranial self-stimulation and sucrose intake differ as hedonic measures following chronic mild stress: interstrain and interindividual differences. Behav Brain Res 2000;107:21-33.
54 Grabe HJ, Lange M, Wolff B, Volzke H, Lucht M, Freyberger HJ, John U, Cascorbi I: Mental and physical distress is modulated by a polymorphism in the 5-HT transporter gene interacting with social stressors and chronic disease burden. Mol Psychiatry 2005;10: 220-224

55 Stroud LR, Salovey P, Epel ES: Sex differences in stress responses: Social rejection versus achievement stress. Biol Psychiatry 2002; 3223

56 Spreckelmeyer KN, Krach S, Kohls G, Rademacher L, Irmak A, Konrad K, Kircher T, Grunder G: Anticipation of monetary and social reward differently activates mesolimbic brain structures in men and women. Soc Cogn Affect Neurosci 2009;4:158-165.

57 Stotland E: The Psychology of Hope. San Francisco, Jossey Bass, 1969.

58 Hammen C: Stress and depression. Annu Rev Clin Psychol 2005;1:293-319.

59 Morris BH, Rottenberg J: Does stress reduce hedonic capacity in anxiety? Poster presented at the 23rd Annual Meeting of the Society for Research in Psychopathology, Minneapolis, 2009.

60 Arai K, Nakagomi Y, Iketani M, Shimura Y, Amemiya S, Ohyama K, Shibasaki T: Functional polymorphisms in the mineralocorticoid receptor and amirolide-sensitive sodium channel genes in a patient with sporadic pseudohypoaldosteronism. Hum Genet 2003;112:91-97.

61 DeRijk RH, Wust S, Meijer OC, Zennaro MC, Federenko IS, Hellhammer DH, Giacchetti G, Vreugdenhil E, Zitman FG, de Kloet ER: A common polymorphism in the mineralocorticoid receptor modulates stress responsiveness. J Clin Endocrinol Metab 2006;91:5083-5089.

-62 Kuningas M, de Rijk RH, Westendorp RG, Jolles J, Slagboom PE, van Heemst D: Mental performance in old age dependent on cortisol and genetic variance in the mineralocorticoid and glucocorticoid receptors. Neuropsychopharmacology 2007;32:1295-1301. 